\title{
DETECTING ANOMALOUS ACTIVITIES IN SPATIO-TEMPORAL REGION
}

\author{
H Manoj T Gadiyar \\ Computer Science and Engineering \\ SDM Institute of Technology Ujire, India \\ Dr. Thyagaraju G S \\ Computer Science and Engineering \\ SDM Institute of Technology Ujire, India \\ Megha Jeevan Kurdekar \\ Computer Science and Engineering \\ SDM Institute of Technology Ujire, India
}

\author{
Kanchana Goudar \\ Computer Science and Engineering \\ SDM Institute of Technology Ujire, India \\ Harshitha K \\ Computer Science and Engineering \\ SDM Institute of Technology Ujire, India \\ Manjushree K \\ Computer Science and Engineering \\ SDM Institute of Technology Ujire, India
}

\begin{abstract}
Anomaly detection system using Machine Learning helps to detect the anomalies like accidents etc. Detecting anomalies in videos is important but it is an unsolved problem. This system will automatically detect the anomaly by image analysis from the surveillance videos. This system is capable of tracking abnormal event in each frame and generates a notification of such event. The proposed K-means algorithm performs existing anomaly detection techniques, while being comparatively time efficient. The system just requires a dataset of video frames and is fed as input to the anomaly detecting module.
\end{abstract}

\section{Keywords-Anomaly Detection, machine learning}

\section{INTRODUCTION}

Today detecting anomalous activities from videos is very challenging due to equivocal nature of anomalies. The important public places such as public garden, super market, university campus, mall etc. these are all under video surveillance. There is very important to provide the security and monitor unusual activities at those places. CCTV has been widely used for monitoring, recording situation in a surveillance system. CCTV cameras are mostly used for a post video forensic process by allowing finding situation from the previous scene. CCTV cameras feed should be manually observed by human beings to find the abnormal event. In this we will be using K-means algorithm to detect the anomalies.

In K-means algorithm number of observation or the dataset are divided into clusters and that clusters are represented as K-clusters. K-clusters have their own feature. K-clusters are put into the group based on the data points. As we know the K-means algorithm is unsupervised learning algorithm.

In our project we will implement software such that it is capable to detecting any anomalies in the video and generate the notification. An object tracking and anomaly detection can be improved by applying machine learning techniques on it.

\section{LITERATURE SURVEY}

Adal Nadjara Toosi and Mohsen Kahani [1] in the year 2010 Soft Computing approach for Anomaly detection. This method used the ANFIS (Adaptive neuro fuzzy interface system) network. This kind of network is a artificial network that applies logical rules to knowledge base to deduce new information It will work on both neural networks and fuzzy logics also. Shekhar R. Gaddam [2] in the year 2017 "Hybrid Anomaly-based Detection Approach". In this method Shekhar R.Gaddam introduced a method which helps to detect anomalies that occurs in an environment by combining the Iterative Dichotomiser3 Decision Tree and Kmeans algorithm. The decision making from a dataset will uses the IterativeDichotomiser 3 decision tree. The Iterative Dichotomiser 3 decision tree begins at the root node S, and when the algorithm reaches the next step from the starting step, it is going to iterate from one variable to another. The Iterative Dichotomiser3 Decision Tree selects the new attribute in the given tree and it calculates the entropy $\mathrm{H}(\mathrm{S})$ or Information gain IG(S).Latifur Khan[3]published in the year 2016 Support Vector Machine based Anomaly Detection. The aim of this paper was to detect anomaly specifically when engaging with large size datasets. In this method they used DGSOT. Yu Guan [4] published on 2013 "Anomaly Detection System using K-means algorithm". Yu Guan was the first person who introduced this method to detect the Anomalies using the K-means algorithm. The different number of observation or the dataset are partitioned into number of clusters in K-means algorithm. The clusters are represented by K- clusters. Each cluster its own features based on the data points that are put in a group. Each cluster has a centroid which is basic element of the clusters; this centroid has some features (information) which separate that particular clusters from other clusters created. The K-means algorithm is unsupervised learning algorithm.

Study carried out on Related Work

The study of the existing systems with respect to 


\section{International Journal of Engineering Applied Sciences and Technology, 2021 Vol. 6, Issue 1, ISSN No. 2455-2143, Pages 441-444 \\ Published Online May 2021 in IJEAST (http://www.ijeast.com)}

the proposed system is carried out referring to the papersmentioned below.

\begin{tabular}{|c|c|c|c|}
\hline $\begin{array}{l}\text { Sl. } \\
\text { No }\end{array}$ & Paper Title & $\begin{array}{c}\text { Techniques } \\
\text { used }\end{array}$ & Application \\
\hline 1. & $\begin{array}{c}\text { Hybrid Deep } \\
\text { Network for } \\
\text { Anomaly } \\
\text { Detection }\end{array}$ & $\begin{array}{c}\text { Hybrid Deep } \\
\text { CNN combining } \\
\text { supervised and } \\
\text { unsupervised } \\
\text { learning } \\
\text { perspective. }\end{array}$ & $\begin{array}{l}\text { Anomaly } \\
\text { detection in } \\
\text { Surveillance } \\
\text { videos of } \\
\text { moving trains, } \\
\text { roads, etc. }\end{array}$ \\
\hline 2. & $\begin{array}{l}\text { Real time } \\
\text { Anomaly } \\
\text { Detection } \\
\text { through CCTV } \\
\text { using Neural } \\
\text { Network }\end{array}$ & $\begin{array}{l}\text { Deep learning } \\
\text { models (CNN } \\
\text { \&RNN) and } \\
\text { algorithm } \\
\text { Automating } \\
\text { Threat } \\
\text { Recognition } \\
\text { System }\end{array}$ & $\begin{array}{l}\text { Automatically } \\
\text { identifies the } \\
\text { irregularities } \\
\text { in normal } \\
\text { patterns of } \\
\text { real time } \\
\text { videos. }\end{array}$ \\
\hline 3. & $\begin{array}{l}\text { Anomaly } \\
\text { Event } \\
\text { Detection in } \\
\text { Security } \\
\text { Surveillanc } \\
\text { e Using 2- } \\
\text { Stream } \\
\text { Based mode }\end{array}$ & $\begin{array}{l}\text { RGB \& } \\
\text { Flow2- } \\
\text { Stream } \\
\text { network of } \\
\text { CNN } \\
\text { assistant }\end{array}$ & $\begin{array}{l}\text { Model utilizes } \\
\text { complementary } \\
\text { information of } \\
\text { 2- Stream } \\
\text { hidden in } \\
\text { Surveillance } \\
\text { video.improve } \\
\text { the performance } \\
\text { of Anomaly } \\
\text { event } \\
\text { detection }\end{array}$ \\
\hline
\end{tabular}

\section{PROPOSED SYSTEM}

Collect the testing video and provide as input to system. This video more feature extracted. With reference to training model testing video classified as normal event and abnormal event.

\section{Input dataset:}

To validate the proposed approach we use dataset which covers relatively more real-world anomaly classes that is standard dataset called UCSD. The UCSD anomaly dataset includes two subsets Ped1and Ped2.We experiment extracted features over this dataset.
Dataset consists of two parts.

- Training set

- Testing set

Both training and testing set contains usual as well as unusual video. The input Surveillance video converted into required format. This video converted to AVI (Audio Video Interleave) format The audio and video data in file container allows synchronous audio with video playback. This video is given as input to system.

\section{Preprocessing:}

Explore the dataset and create the training and testing set. Then employ the training set to teach the model and testing set to estimate the trained model .video frames using the function 'NumberIffFrames'.These video frame is a one of many still images which create the complete moving picture. The video frames undergo segmentation. The segmentation is positioning video sequence into disjoint sets of sequential frames that are homogeneous according The input videos are read by system using function 'VideoFileReader'.Then that video is converted into to some defined criteria. Drawing out frames from all the input videos in the training as well as the testing set . Preprocessing these frames and then teaching a model utilizing the frames in the training set. Evaluation of the model using the frames present in the testing set.

\section{K-means algorithm:}

The Surveillance video is categorized as normal and abnormal event by K-means algorithm. It is an unsupervised algorithm that works by creating clusters from the training dataset. The number of cluster centroids indicated by ' $\mathrm{k}$ '. The algorithm works by randomly initializing $\mathrm{k}$-centroids. Then, Euclidean distance is computed between each cluster centroid and each training samples in the dataset. Each data point is allotted to its closest cluster centroid. At the final step of the first iteration, all of the training samples K-means continue are categorized under those $\mathrm{k}$ centroids. Then the centroid values are updated as the mean of all its contents. This algorithm will iteratively compute Euclidean distances, assign points to clusters and update clusters. It continues until there is no significant change in the cluster centroid values and its contents. For a new instance, Euclidean distance will be computed between all cluster centroids and the instance, and it will be allotted the tag of the nearest centroid. In our experiment there are two labels normal event and abnormal event. All detected events are assigned to their respective labels. 
International Journal of Engineering Applied Sciences and Technology, 2021

Vol. 6, Issue 1, ISSN No. 2455-2143, Pages 441-444

Published Online May 2021 in IJEAST (http://www.ijeast.com)

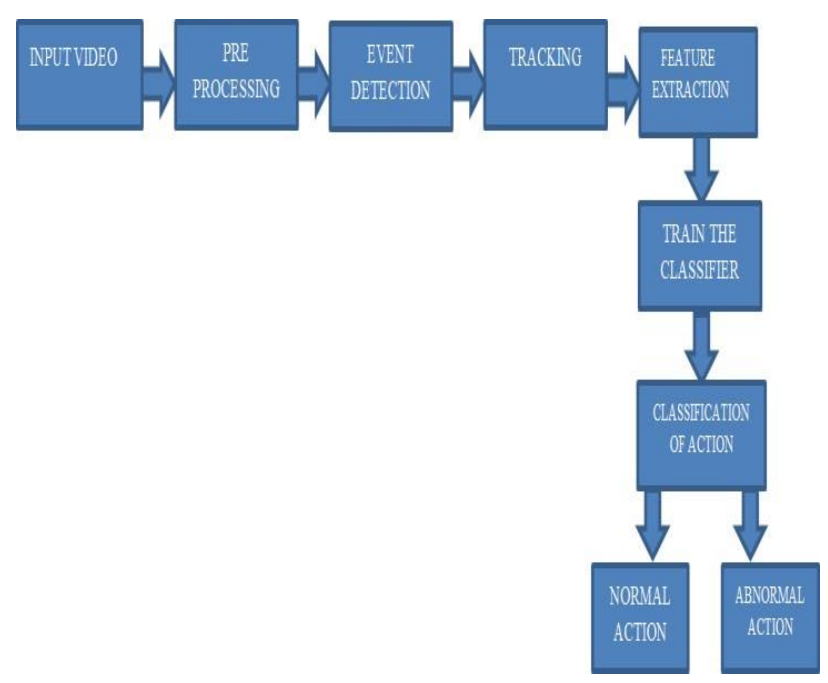

\section{USER INTERFACE DESIGN}

The user interface design of this proposed system is shown below:

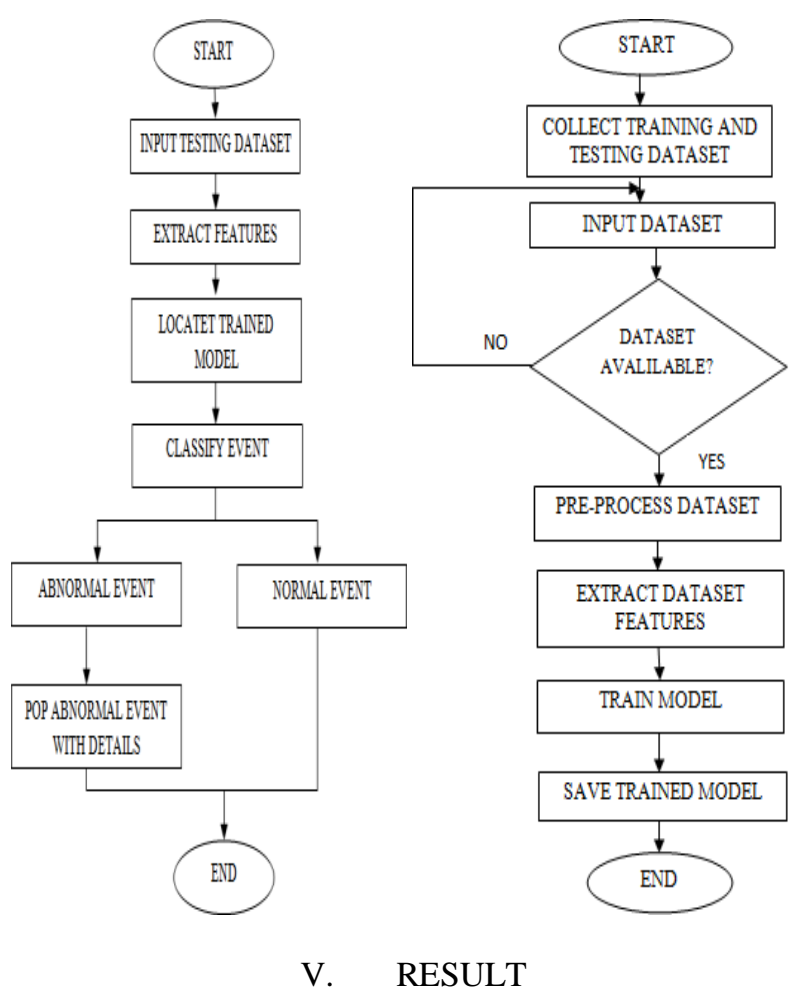

The input video UCSD dataset is provided to this system. The feature extraction is done and displays number of frames imported. Then it shows number of feature generated and length of each frame. If no anomalous activities are detected then it prints normal. Or else if any anomalous activities are detected then it displays at the time from where the abnormal event is detected and also shows pop-up video clip of that event.

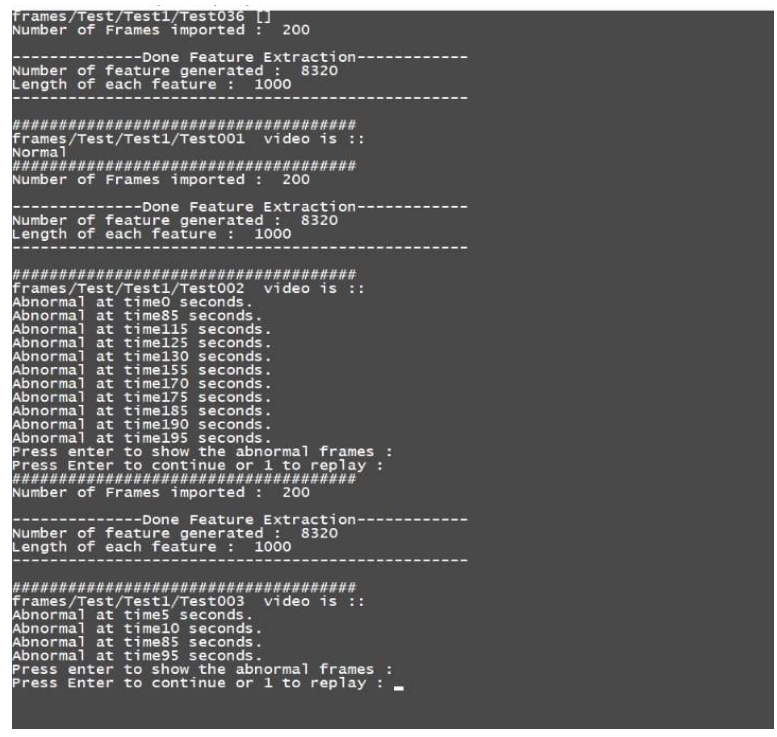

\section{Figure 1: Command prompt user interface}

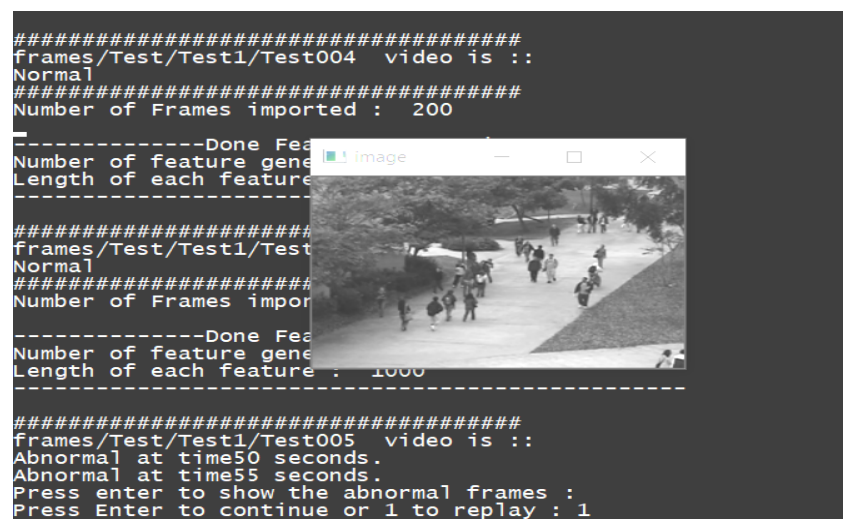

Figure 2: Abnormal event video frame.

$$
\text { VI. CONCLUSION }
$$

We have introduced an unsupervised algorithm for anomaly detection that is suitable for analyzing large multifaceted time-series and can detect anomalous regions not only in temporal but also in spatio-temporal data from various domains.

The proposed MDI algorithm surpass existing anomaly detection techniques, while being comparatively time efficient; Thanks to an productive implementation and a novel interval proposal technique that excludes uninteresting parts of the data from in- depth analysis.

\section{REFERENCES}

[1] Tadashi Ogino, "Detection System for Video data Using Machine Learning", International Journal of Knowledge Engineering, Volume 2, No. 2, June, 2016.

[2] B.V Emmanu Varghese, Jaison Mulerikkal and Smitha Mathew, "Video Anomaly Detection in 
Confined Areas", 2017.

[3] Amuthan prabakar Muniyandi, R. Rajeswari and R. Rajaram, "Network Anomaly Detection by the Cascading K-Means Clustering algorithm and C4.5 Decision Tree algorithm", International Conference on Communication Technology and System Design (ICCTSD), Volume 30, pp. 174-182, May 2012.

[4] Sandhya Rani Sahoo, Ratnakar Dash, Ramesh $\mathrm{Ku}$. Mahapatra and Baishnabi Sahu, "Unusual Event Detection in Surveillance Video using Transfer Learning", International Conference on Information Technology (ICIT), :IEEE, 2019.

[5] Jinghua Wang and Guoyan Zhang, "Video Data Mining based on K-means Algorithm for Surveillance Video", Computer Science and Technology Department, Hua Zhong Normal University :IEEE, 2011.

[6] K. Meena, A. Viji, J. Joshan Athanesious and V. Vaidehi, "Detecting Abnormal Event in Traffic Scenes using Unsupervised Deep Learning Approach", Department of Electronics, Madras
Institute of Technology :IEEE, July 2020.

[7] Aiswarya Mohan, Meghavi Choksi and Mukesh A Zaveri, "Anomaly and Activity Recognition Using Machine Learning Approach for Video Based Surveillance", 10th International Conference, Communication and Networking Technologies (ICCNT) :IEEE, 2019.

[8] Nguyen Thanh Van and Tran Ngoc Thinh, "An anomaly based network intrusion detection system using deep learning", International Conference on System Science and Engineering (ICSSE), pp. 210-214 :IEEE,2017.

[9] Claudio Piciarelli, Christian Micheloni and Gian Luca Foresti, "Trajectory Based Anomalous Event Detection", IEEE Transaction on Circuits and Systems for Technology ,Volume 17, No. 11, November 2008.

[10] Adal Nadjara Toosi and Mohsen Kahani, "Support Vector Machine based Anomaly Detection", 2016. 